\title{
Leucocytosis and type 2 diabetes mellitus - a case report
}

\section{Background}

Type 2 Diabetes Mellitus is the most common form of diabetes among middle-age population, with an increase in newly diagnosed cases in the past several years due to multiple factors: hereditary legacy, changes in lifestyle and nutrition, lack of physical activity. The following article is focused on the case of a recent diagnosed case of type 2 Diabetes Mellitus in a patient with multiple co morbidities and an atypical presentation at the diabetes clinic.

\section{Method and results}

The patient is a 54-year old male, former heavy smoker and with a history of alcohol consumption, which presented for high glycaemic values, fatigue, and fever, diahreea and weight loss. The patient has no family history of diabetes, but major cardiovascular risk and bilateral thigh amputation due to thromboangiitis obliterans in the past three years, after undertaking a lombar sympathectomy with no amelioration of pain or claudication.

During the admission, the patients' condition seemed to improve, altough the white blood count remained at high levels all that time. There was only one episode of fever, the highest temperature reaching $37^{\circ} \mathrm{C}$, point at which hemoculture was collected. The fever was remitted after administrating antibiotics. Basal bolus insulin therapy was initiated with good results regarding the patients' glycaemic levels. Laboratory parameters evidenced leukocytosis syndrome with white blood cells over $30000 / \mathrm{mm}^{3}$, inflammatory syndrome, high values of conjugated bilirubin, hyperglycemia, mild anemia. Subsequently, hemo and uroculture were proven negative, as well as stool samples for Salmonella, Shigella, and Yersinia. Imagistic analysis were also performed, abdominal ultrasound revealed increased size of the liver, inflammation of the gall bladder without gallstones and as cites fluid with perihepatic, perisplenic and pelvic disposition. Abdominal MRI confirmed the ultrasound results.

\section{Discussions}

The main challenge in the therapeutic management of this case was the persistence of leukocytosis, in the absence of any proof of viral, bacterial or fungal infection or other factors for inflammation. The high value of white blood cells, along with neutrophilia usually indicates an infection, but this theory is not supported by paraclinical results.

Differential diagnosis took into consideration a hematological pathology like leukemia, multiple myeloma, but also hepatic cytolysis and cholestasis syndrome. In the absence of a blood smear and targeted investigations, a hematological disease cannot be excluded. Nevertheless, leukemia should associate with normochromic, normocytic anaemia, thrombocytopenia and blastic cells in the blood smear. Also, the diagnosis of multiple myeloma cannot be confirmed in the absence of hypercalcemia, elevated serum creatinine.

Hepatic cytolysis could not be confirmed with a value of AST/ALT ratio of 1,68 , with should be higher than 2 .
Volume 5 Issue 5 - 2018

\author{
Ciprian Constantin,' Tudor Veronica \\ Gabriela $^{2}$ \\ 'Central Military Hospital, Bucharest, Romania \\ 'Elias" Emergency University Hospital, Bucharest, Romania
}

Correspondence: Ciprian Constantin, Central Military Hospital, Bucharest, ROMANIA Tel + 40 731040974,

Email ciprian_constantin@yahoo.com

Received: July 31, 2018 | Published: October 04, 2018

The diagnosis for cholestasis syndrome is sustained only because of the high values of conjugated bilirubin, but it is not enough in the absence of abnormal values of alkaline phosphatase and gamma glutamyl transferase.

One of the theories regarding the gastrointestinal manifestations for this patient is an intestinal manifestation of Buerger's Disease. Buerger's disease, also called thromboangiitis obliterans (TAO), is a recurrent vaso-occlusive disease. Thromboangiitis obliterans (TAO) is a nonatherosclerotic, segmental inflammatory disease that most commonly affects the small and medium-sized arteries and veins in the upper and lower extremities. ${ }^{1}$ in the characteristic acute-phase lesion, in association with occlusive cellular thrombosis, the acute inflammation involving all layers of the vessel wall led TAO to be classified as a vasculitis. Typically, the disease affects middle-aged male smokers and involves small and medium-sized arteries, veins and nerves of the upper and lower extremities. ${ }^{2}$ Systemic manifestations involving mesenteric, cerebral, and coronary arteries are exceptional and multisystem involvement of two or more organs is extremely rare. The disease usually affects medium and small arteries and veins of the upper and lower extremities. Visceral artery involvement is rarely reported. Use or exposure to tobacco plays a central role in the initiation and progression of the disease, which is consistent with the patient's history and could explain the chronic inflammatory syndrome. ${ }^{3}$

Sympathectomy may be helpful in pain relief as a short term solution, but this method has not proved to have any benefits in treating the disease. ${ }^{4}$

Aspirin and clopidogrel may be efficient in preventing secondary events, but here is no evidence, however, to suggest that the symptoms of claudication are reduced by long-term treatment. ${ }^{5}$

Abstinence from smoking is the only definitive treatment to prevent disease progression. Medical line of treatment with vasodilators, pentoxyfylline, and cilostazol may help improve pain-free walking distance but cannot prevent disease progression. Early diagnosis and aggressive therapy can decrease patient symptoms and chances of major amputations. 


\section{Beyond conclusion}

Leukocytosis is the key element around which the differential diagnosis has been constructed here and this can be analyzed in the context of chronic alithiasis cholecystitis syndrome, but also as part of the paraclinic panel of Thromboangiitis obliterans (Buerger Disease) in a former heavy smoker patient. A possible haematological pathology cannot be excluded in the absence of targeted investigations. During this hour when this article was written we could confirm that the patient is home, with a regular lifestyle, with a chronic treatment presented here and - in the same time - with the same question adressed to medical system: is it my leukocitosis a real challenge if my clinical appearance is OK?

Post scriptum: While no tests can confirm whether you have Buerger's disease, your doctor will likely order tests to rule out other more common conditions or confirm suspicion of Buerger's disease brought on by your signs and symptoms. ${ }^{6}$ Tests may include: Blood test, The Allen's test, Angiogram (Mayo Clinic). ${ }^{7}$

\section{Acknowledgments}

None.

\section{Conflict of interest}

The author declares that there is no conflict interest.

\section{References}

1. Iwai $\mathrm{T}$, Inoue $\mathrm{Y}$, Umeda $\mathrm{M}$, Et al. Oral bacteria in the occluded arteries of patients with Buerger disease. J Vasc Surg. 2005;42(1):107-115.

2. Olin JW. Thromboangiitis obliterans (Buerger's disease). N Engl J Med. 2000;343(12):864-869.

3. Adar R, Papa MZ, Halpern Z, et al. Cellular sensitivity to collagen in thromboangiitis obliterans. N Engl J Med. 1983;308(19):1113-1116.

4. Magalhães Ede P, Trevisan M, Mochizuki M, et al. Intestinal ischemia as a single manifestation of thromboangiitis obliterans: a case report. Angiology. 2005;56(6):789-792.

5. Krupski WC, Selzman CH, Whitehill TA. Unusual causes of mesenteric ischemia. Surg Clin North Am. 1997;77(2):471-502.

6. Kobayashi M, Kurose K, Kobata T, et al. Ischemic intestinal involvement in a patient with Buerger disease: case report and literature review. J Vasc Surg. 2003;38(1):170-174.

7. Borlaza GS, Rapp R, Weatherbee L, et al. Visceral angiographic manifestation of thromboangiitis obliterans. South Med J. 1979;72(12):1609-1611. 